\title{
Community Health Nurse Specialist
}

National Cancer Institute

\section{Source}

National Cancer Institute. Community Health Nurse Specialist. NCI Thesaurus. Code C20834.

A registered nurse prepared at the master's degree level or higher who specializes in exploring and meeting the needs of select populations in community contexts. (UMD, SON) 